\title{
Tracking Circadian Rhythms of Bone Mineral Deposition in Murine Calvarial Organ Cultures
}

\author{
John-David P McElderry, ${ }^{1}$ Guisheng Zhao, ${ }^{2}$ Alexander Khmaladze, ${ }^{1}$ Christopher G Wilson, ${ }^{2}$ \\ Renny T Franceschi, ${ }^{2}$ and Michael D Morris ${ }^{1}$ \\ ${ }^{1}$ Department of Chemistry, University of Michigan, Ann Arbor, MI, USA \\ ${ }^{2}$ School of Dentistry, University of Michigan, Ann Arbor, MI, USA
}

\begin{abstract}
Osteoblasts, which orchestrate the deposition of small apatite crystals through the expression of nucleating proteins, have been shown to also express clock genes associated with the circadian signaling pathway. We hypothesized that protein-mediated bone mineralization may be linked to circadian oscillator mechanisms functioning in peripheral bone tissue. In this study, Per1 expression in ex vivo neonatal murine calvaria organ cultures was monitored for 6 days using a Per1-luciferase transgene as a bioluminescent indicator of clock function. Fluctuations in Per1 expression had a period of $25 \pm 4$ hours $(n=14)$ with early expression at CT09:59 $\pm 03: 37$ (CT = circadian time). We also established the kinetics of mineral deposition in developing bone by using noninvasive Raman microscopy to track mineral accumulation in calvarial tissue. The content and quality of newly deposited mineral was continually examined at the interparietal bone/fontanel boundary for a period of 6 days with 1-hour temporal resolution. Using this approach, mineralization over time exhibited bursts of mineral deposition followed by little or no deposition, which was recurrent with a periodicity of $26.8 \pm 9.6$ hours. As many as six near-daily mineralization events were observed in the calvaria before deposition ceased. Earliest mineralization events occurred at CT16:51 $\pm 03: 45$, which is 6 hours behind Per1 expression. These findings are consistent with the hypothesis that mineralization in developing bone tissue is regulated by a local circadian oscillator mechanism. ( 2013 American Society for Bone and Mineral Research.
\end{abstract}

KEY WORDS: CIRCADIAN RHYTHM; RAMAN SPECTROSCOPY; OSSIFICATION; APATITE; MINERALIZATION; CRYSTALLINITY

\section{Introduction}

$\mathrm{M}$ ammalian circadian rhythms are controlled by a negative feedback mechanisms involving clock genes. A circadian system functions in three parts: (1) a timing cue, (2) a circadian oscillator, and (3) a rhythmic physiological response. ${ }^{(1)}$ The master regulator for presumably all circadian rhythms in the body has been identified as the suprachiasmatic nucleus (SCN), located in the hypothalamus. ${ }^{(2)}$ The SCN receives timing cues from retinal nerves sensitive to day/night light-level fluctuations and responds by sending rhythmic neural and humoral signals to the body. ${ }^{(3-6)}$ Circadian oscillators also reside in peripheral tissues, where they receive timing cues from the SCN and direct the rhythmic expression of other downstream genes. Mammalian circadian oscillators consist of a nuclear complex composed of three PERIOD (PER1, PER2, and PER3) and two Cryptochrome (CRY1 and CRY2) proteins that are activated by the CLOCK:BMAL heterodimeric transcription factor. ${ }^{(7)}$ Once induced, the PER/CRY complex suppresses CLOCK:BMAL transcriptional activity, thereby halting the production of PER and CRY proteins. ${ }^{(8)}$ With the turnover of PER/CRY, the CLOCK:BMAL complex is reactivated to start a new cycle. ${ }^{(9)}$

Musculoskeletal tissues, including vertebral bone, ${ }^{(10)}$ craniofacial bone, ${ }^{(11,12)}$ isolated osteoblasts, ${ }^{(13)}$ and skeletal muscle, ${ }^{(14)}$ all exhibit circadian expression of Per and Cry genes. Although peripheral circadian oscillators have been implicated in the physiological functions of various tissues, ${ }^{(15-19)}$ the extent of their influence is unknown. CLOCK:BMAL transcribes a variety of downstream targets, collectively termed clock-controlled genes (CCGs). CCGs that encode other transcription factors amplify the number of cycling genes and are able to influence other cells, tissues, and extracellular processes. ${ }^{(20)}$ Secondary negative feedback loops created by CCGs are capable of modifying the downstream circadian effect in phase and amplitude. Therefore, the circadian network has the potential to influence a physiological pathway at several points. ${ }^{(21)}$ In bone tissue, the

Received in original form December 3, 2012; revised form February 18, 2013; accepted March 6, 2013. Accepted manuscript online March 16, 2013. Address correspondence to: Michael D Morris, PhD, Department of Chemistry, University of Michigan, 930 N. University Ave., Ann Arbor, MI 48109-1055, USA. E-mail: mdmorris@umich.edu

Journal of Bone and Mineral Research, Vol. 28, No. 8, August 2013, pp 1846-1854 DOI: $10.1002 / j b m r .1924$

(C) 2013 American Society for Bone and Mineral Research 
role of circadian oscillators has been implicated in bone turnover, humoral signaling, and fracture healing. ${ }^{(22-24)}$ Furthermore, deletion of Per1, Per2, and Bmal1 genes increases bone mass. ${ }^{(13)}$ However, the relationship between clock gene function and mineralization remains a relatively new and little explored research topic. Understanding the relationship between bone cell function and the circadian network may be integral to understanding the physiological process of ossification.

Bone mineralization is a protein-mediated process. The mechanistic steps required for calcium phosphate mineral formation are thought to be regulated by several genes including Bsp (bone sialoprotein), Dmp1 (dentin matrix protein 1), Bglap2 (osteocalcin), and Spp1 (osteopontin). Each of these proteins can directly interact with growing mineral using domains containing repeated acidic residues. These proteins may function as either nucleators or inhibitors to mineralization. ${ }^{(25)}$ However, the mechanisms of bone mineralization are currently not well understood, and as the intricacies of the mineralization process are elucidated a clearer understanding of protein function will evolve. ${ }^{(26,27)}$

Modern analytical techniques for studying mineralization in situ are revealing more specific roles for these proteins. Nudelman and colleagues ${ }^{(28)}$ proposed that uptake of calcium phosphate clusters into the collagen fibril is regulated by tuning the electrostatics of the clusters with negatively charged protein segments. These protein segments are then directed to binding sites within the gap regions, where the calcium phosphate clusters nucleate into apatitic mineral. Some investigators suggest that mineral does not form an apatitic phase directly, but rather passes through an intermediate solid phase stabilized by nucleating proteins. ${ }^{(28-30)}$ The existence of this intermediate is currently debated. ${ }^{(30,31)}$

We hypothesize that bone mineralization is linked in part to regulated protein expression by peripheral oscillators within the circadian network. Identifying a link between these two physiological processes may have clinical relevance, because the search for more effective anabolic agents relies heavily on known mechanisms of ossification. The present study was designed to determine, with high temporal resolution, the kinetics of mineral activity by continuously monitoring mineral deposition in neonatal murine calvarial tissue using Raman microscopy. Here we report that mineral deposition in calvarial organ cultures shows an approximately 24-hour periodicity and can be correlated with clock gene activity.

\section{Materials and Methods}

\section{Specimens}

All procedures involving mice conformed to an Institutional Animal Care and Use Committee-approved protocol. Colonies of C57/Bl6 mice were purchased from Charles River Laboratories (Wilmington, MA, USA). B6.BTBR-Tg(Per1-luc,Per1)1Jt/J mice, a transgenic line that harbors firefly luciferase under the control of a 6.75-kb Per1 promoter (Per1-Luc mice), ${ }^{(32)}$ were purchased from The Jackson Laboratory (Bar Harbor, ME, USA). All mice were housed in University of Michigan animal facilities and subjected to a 12/12-hour light/dark (LD) cycle throughout gestation. Within 24 hours of birth, neonatal mice were collected and sacrificed. Calvaria including frontal, parietal, and interparietal plates were harvested with brain and soft tissue removed. The fontanel region remained intact. Circadian time (CT) was defined by the LD cycle used before sacrifice, with CT00:00 and CT12:00 corresponding to the beginning and end of the light period. Time of sacrifice was consistently CT05:00 regardless of the time of birth. This timing convention was used postmortem.

\section{Ex vivo organ culture}

During tissue preparation, calvaria were cultured in Dulbecco's Modified Eagle's Medium (DMEM, which contains $1.8 \mathrm{mM} \mathrm{Ca}^{+2}$, $\left.0.916 \mathrm{mM} \mathrm{PO}_{4}{ }^{-3}\right)$ under standard growth conditions $\left(5 \% \mathrm{CO}_{2}, 37^{\circ} \mathrm{C}\right.$, $90 \%$ humidity). Six-well cell culture dishes (In Vitro Scientific, Sunnyvale, CA, USA) fitted with \#1 glass windows in the bottom of each well were used in all experiments. Sapphire flats (12-mmdiameter, 1-mm-thick; Esco Products, Inc., Oak Ridge, NJ, USA) were placed on top of the glass windows. Calvaria were placed on the sapphire, brain-side up, with cuts made radially with a scalpel to allow for flattening of the tissue. A 316-stainless-steel mesh screen and washer (Small Parts Inc., Seattle, WA, USA) were placed on top of the calvaria for 4 hours until tissue remained flat, and then removed. Tissue was then stained with alizarin red $(1 \mu \mathrm{g} / \mathrm{mL})$ for 30 minutes and then washed in growth medium. In each well, a custom tissue immobilizer device made with 316stainless-steel was used to anchor the specimen at a single point (Fig. 1A, B). The growth medium was removed and differentiation medium containing DMEM, 10\% fetal bovine serum, $100 \mu \mathrm{g} / \mathrm{mL}$ streptomycin, 100 units $/ \mathrm{mL}$ penicillin, $100 \mu \mathrm{g} / \mathrm{mL}$ ascorbic acid phosphate, and $10 \mathrm{mM} \beta$-glycerol phosphate was used throughout the rest of the experiment. ${ }^{(33)}$ The addition of differentiation medium marked time-zero for time-series experiments. Medium for Per1-luc murine calvaria was supplemented with $50 \mu \mathrm{g} / \mathrm{mL}$ luciferin for bioluminescence experiments. LD cycling was replaced with continuous dark (DD) for all organ cultures and maintained throughout the experiments.

\section{RNA isolation and RT-PCR}

Total RNA was isolated using Trizol reagent (Invitrogen). Two micrograms of total RNA was reverse transcribed using TaqMan reverse transcriptase (Applied Biosystems, Foster City, CA, USA) for CDNA synthesis. Quantitative real-time polymerase chain reaction (PCR) detection of Per1, Per2, Dbp, and Clock mRNAs was carried out as described previously ${ }^{(34)}$ using predeveloped TaqMan probes and an ABI PRISM 7700 sequence detector (Applied Biosystems). The mRNA expression for each gene was calculated based on a relative standard curve and normalized to glyceraldehyde-3-phosphate dehydrogenase mRNA (GAPDH).

\section{Raman instrumentation}

Mineral deposition in C57/Bl6 murine calvarial tissue was observed using a custom-built Raman inverted microscope. The Raman microscope consisted of an inverted microscope (Eclipse Ti; Nikon USA, Melville, NY, USA) with the beam-shaping lenses removed from the rear port. Collimated 830-nm laser light was directed through the rear port and focused on the specimen through an infinity-corrected $20 \times / 0.45$ numerical aperture (NA) objective (Nikon USA). Raman scattered light was collected 


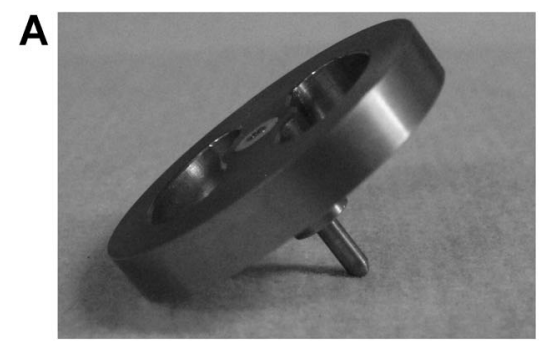

B

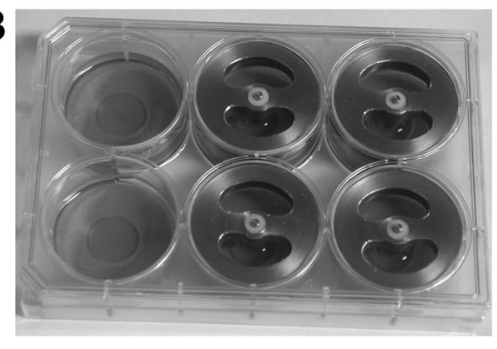

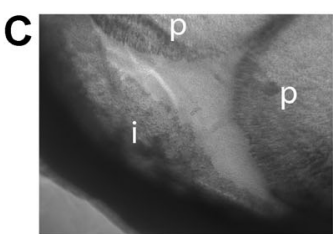
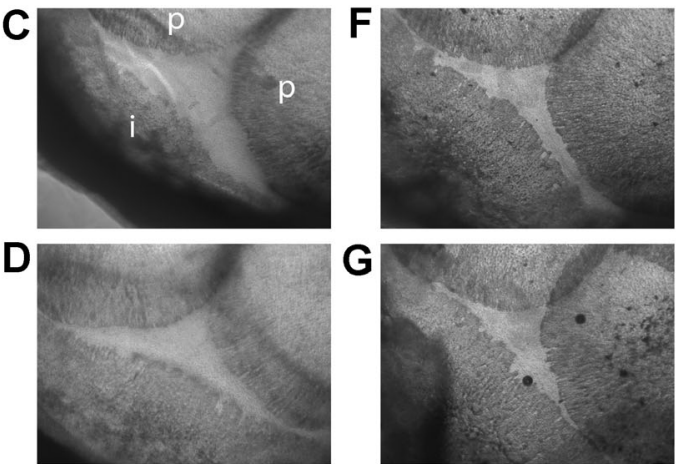

$\mathrm{E}$

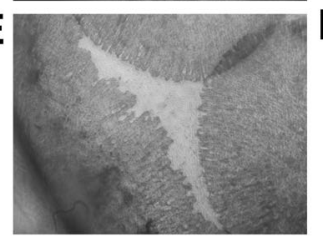

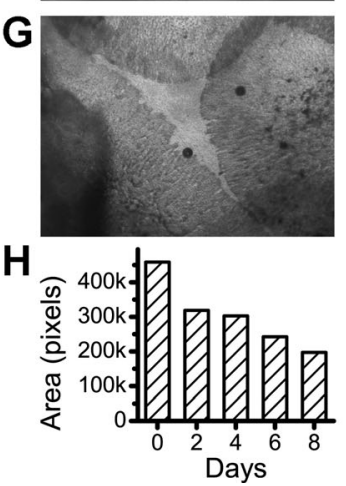
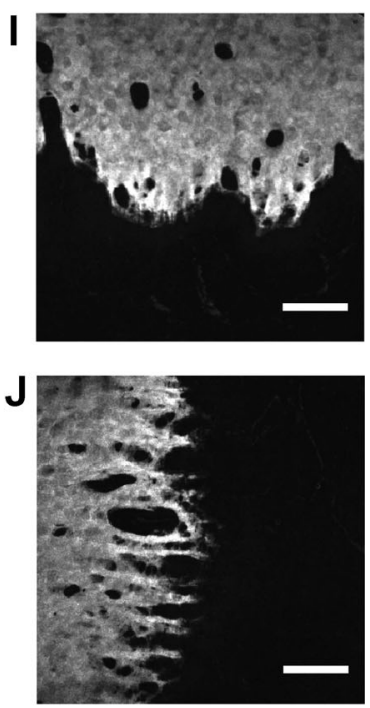

Fig. 1. Ex vivo murine calvarial organ culture. (A) Tissue immobilizing device used to hold the interparietal bone by a single point in a six-well plate (plate shown in $B$ ). White light images of alizarin-stained calvarial tissue showing posterior fontanel closure after $(C) 0,(D) 2,(E) 4,(F) 6$, and $(G) 8$ days of culture. Labels $i$ and $p$ in $C$ indicate interparietal and parietal bones. $(H)$ Bar chart shows quantitative results of fontanel area measured in image pixels over 8 days. Confocal fluorescence images of alizarin-stained calvaria at the $(I)$ interparietal and $(J)$ parietal bone edge with the bar showing $100-\mu \mathrm{m}$ scale.

through the rear port and focused through a $50-\mu \mathrm{m}$ slit into a spectrograph (HoloSpec; Kaiser Optical Systems, Inc., Ann Arbor, MI, USA) using a 200-mm achromatic tube lens and ultimately imaged using a back-illuminated deep-depletion charge-coupled device (CCD) (Classic CCD; Andor Technologies, South Windsor, CT, USA). Spectral resolution was 6 to $8 \mathrm{~cm}^{-1}$. By digitally resolving spectral bands, differences in wave number shift of $<1 \mathrm{~cm}^{-1}$ could be detected. Digital resolution of spectral bands in the 900 to $1010 \mathrm{~cm}^{-1}$ region was performed by band fitting the spectra to Gaussian + Lorentzian functions using a nonlinear least-squares technique described in Press and colleagues ${ }^{(35)}$ and implemented in MATLAB (Math Works, Natick, MA, USA). ${ }^{(36)}$

Organ cultures containing calvaria and medium were maintained under standard culture conditions throughout the experiment in a stage incubation chamber (LiveCell; Pathology Devices, Inc., Westminster, MD, USA). The microscope was fitted with a computer-controlled $x-y-z$ stage (Proscan II; Prior Scientific, Rockland, MA, USA) with $<1 \mu \mathrm{m}$ spatial resolution. Raman spectra were taken simultaneously on up to four calvaria by interrogating each specimen every hour for 120 to 140 hours.

Calvaria were positioned so that the laser line illuminated the interparietal bone/fontanel interface perpendicular to the sagittal plane. The quantity of mineral deposition in bone collagen was measured as the phosphate $v_{1}$ Raman band height at $957 \mathrm{~cm}^{-1}$ using the phenylalanine band height at $1001 \mathrm{~cm}^{-1}$ as an internal measure of matrix content. Mineralization rates were determined by applying a quadratic least-squares time derivative (Savitzky-Golay, 13 point) of the mineral-to-matrix data. The crystalline phase of the mineral was measured using the phosphate $v_{1}$ band position. Mineral with a band centered at 957 to $960 \mathrm{~cm}^{-1}$ was considered to be apatitic. Crystallinity, a combined measure of crystal size and perfection, was measured as the inverse of the phosphate $v_{1}$ band width (at $957 \mathrm{~cm}^{-1}$ ). Crystallinity is measured in units of nanometers $(\mathrm{nm})$, corresponding to crystal domain size in the c-axis; however, because of lattice substitutions distorting the structure, this measure is best interpreted as a lower limit to domain size. The actual crystallite size may be larger.

\section{Bioluminescence imaging}

Bioluminescence of Luc gene coexpression in transgenic murine calvaria was followed with an ultralow noise electron multiplying CCD (EMCCD) (iXon, $512 \times 512$ pixels; Andor Technologies, Windsor, CT, USA) with $16 \times 16$ pixel binning, attached to one port of the microscope. $A \times 0.5$ magnification relay optic was used to reduce the image to increase the amount of light per pixel. Tissue was imaged using a $10 \times 0.5 \mathrm{NA}$ objective (Nikon USA) with 14-minute acquisition. Fluorescence images of alizarin-stained calvaria were also performed with the same EMCCD channel with the addition of a mercury lamp excitation source and a filter cube (TRITC-A-NTE; Semrock, Rochester, NY, USA). The Raman illumination region was determined by imaging the laser spot on the tissue.

\section{Results}

Bone formation was observed in an ex vivo tissue culture system, which allowed examination of active mineralization areas in neonatal calvaria over periods of up to a week. Mineralization activity in this model was initially verified by culturing calvarial tissue for $0,2,4,6$, and 8 days, followed by staining with alizarin red and fluorescence imaging of the fontanel region. Analysis of the calvarial images in Fig. $1 C-G$ showed that the integrated area of the posterior fontanel decreased continually over 8 days (summarized in Fig. $1 H$ ). There was no mineral detected within the fontanel region to suggest nonspecific mineralization of the tissue. However, mineral deposition was confined to the fontanel perimeter, which was observed over time as an inward moving bone edge, as would be expected of an advancing mineral front along the interparietal and parietal bone edges. The pattern of 
bone formation at the parietal bone edge was highly porous and divided, whereas the interparietal bone edge was more uniform (Fig. $1 /$ and $J$ ). We limited our study to mineralization occurring at the interparietal bone edge.

\section{Detection of clock gene activity in isolated calvarial organ cultures}

To determine if clock genes were active in calvarial organ cultures, we measured mRNA levels of Period1 (Per1), Period2 (Per2), Clock (Clk), and D-box binding protein (DBP) in isolated tissues. All four transcripts were detected, although we did not attempt to directly measure if levels varied in a circadian pattern (Fig. 2A). In several tissues, circadian oscillators continue to function even when the tissue has been put in organ culture and removed from central SCN regulation. ${ }^{(32)}$ To determine if the circadian oscillator in calvaria could function in organ cultures, we isolated calvaria from B6.BTBR-Tg(Per1-luc, Per1)1 Jt/J transgenic mice. Dual-channel images using fluorescence and bioluminescence techniques provided spatial maps for relative Per1 expression levels in the posterior fontanel region (Fig. 2B). Calvaria were stained with alizarin red before culturing to show clear mineralization boundaries of the two parietal bones and the interparietal bone surrounding the posterior fontanel and imaged with the fluorescence channel. Bioluminescence images were taken immediately afterward, with no excitation source. Spatial maps of six Per1-Luc calvarial tissue sections at $T=0$ showed ubiquitous expression of the luciferase reporter in the calvaria, whereas three wild-type controls showed baseline bioluminescence. Per1 expression was highest within the fontanel region. There was a significant reduction in luciferase activity at the interparietal bone edge (indicated as black line in Fig. $2 B$ ) with only moderate expression inside the bone region.

Bioluminescence from Per1-Luc was monitored longitudinally in 14 ex vivo calvarial cultures for 6 days. Time series data of the integrated signal showed peak expression of Per 1 in nine of the specimens with periodicity persisting for up to 6 days. These specimens had periods ranging from 18 hours to 28 hours with an average period of $25 \pm 4$ hours. An example of Per1-Luc bioluminescence over time is shown in Fig. 2C. Per1 expression in this specimen has a 27-hour period starting in the middle of the light period (СT07:26, circadian time) and gradually shifting to
A

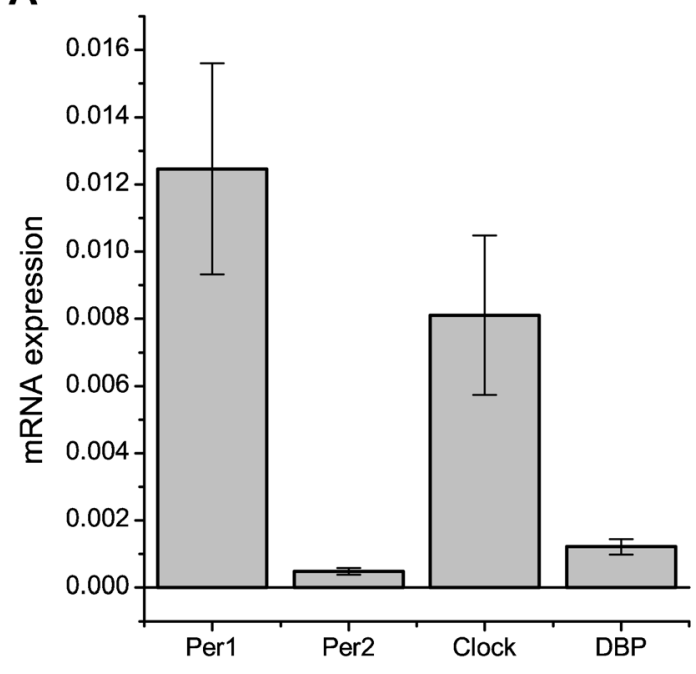

B Alizarin Fluorescence
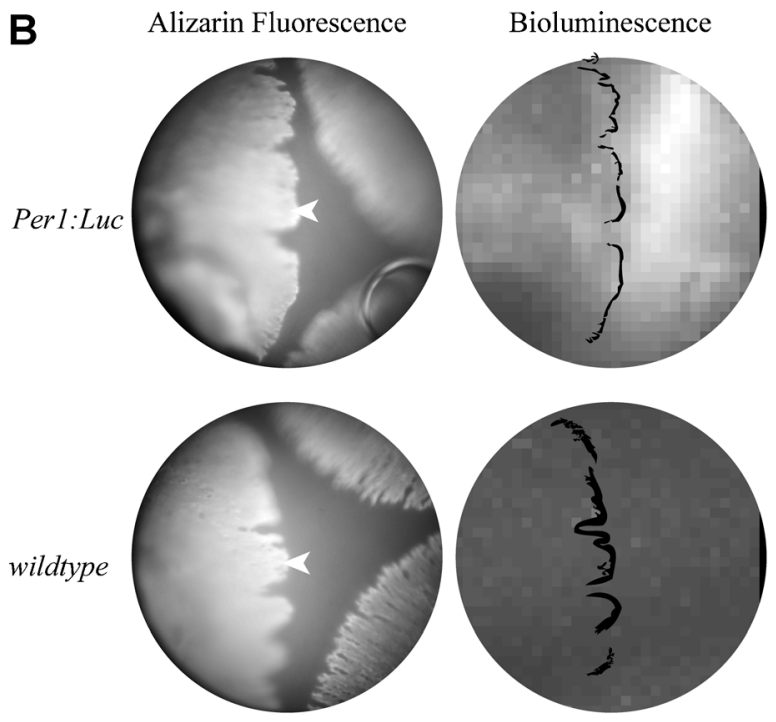

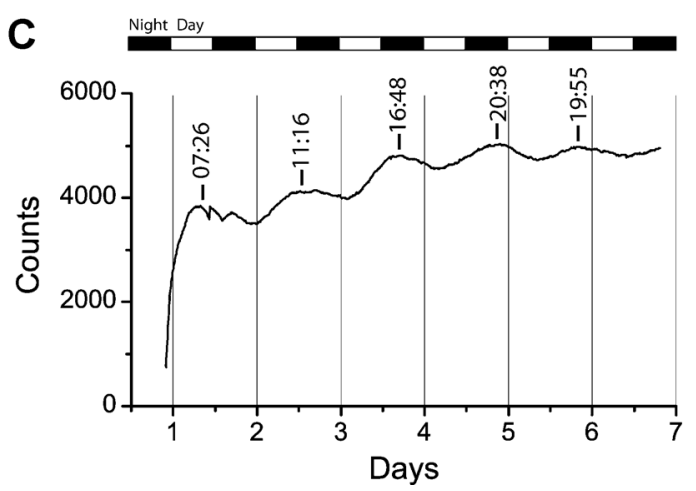

Fig. 2. Clock gene expression in ex vivo calvarium culture. (A) Reverse-transcriptase PCR measurements of mRNA expression of Per1, Per2, Clock, and DBP from calvarial tissue cultures, normalized to GAPDH. (B) Fluorescence and bioluminescence images of Per1:Luc and wild-type calvarial tissue sections at $\mathrm{T}=0$. Fluorescence and bioluminescence images are shown in grayscale with white representing mineralized tissue and Per1 expression, respectively. Black lines represent interparietal bone edge. White arrowheads indicate placement of Raman probe. (C) Bioluminescence intensity for Per1:Luc calvarial sections in circadian time. Black and white bars indicate dark and light times during gestation. 
the middle of the dark period (CT19:55) over the 6-day observation. Five calvaria did not show continued expression beyond one cycle and therefore were not useful for measuring periodicity.

\section{Measurement of calvarial mineralization by Raman spectroscopy}

Active mineral deposition in regions of new bone formation was observed by Raman microscopy. Figure $3 A$ shows time-series Raman spectra observed near the interparietal bone edge of a calvarium section. The $\mathrm{PO}_{4}{ }^{3-}$ band at $957 \mathrm{~cm}^{-1}$ is characteristic of newly deposited apatitic minerals and increased over 6 days. The amino acid residues phenylalanine (Phe, $1001 \mathrm{~cm}^{-1}$ ), proline (Pro, 850 and $925 \mathrm{~cm}^{-1}$ ), and hydroxyproline (Hyp, 875 and 940 $\mathrm{cm}^{-1}$ ), which are components of the organic matrix, showed little variation and no significant change in intensity throughout the experiment. Therefore, tissue mineral content could be measured as the ratio of the $\mathrm{PO}_{4}{ }^{3-}$ band height to the Phe band height (mineral-to-matrix). Similar time-series plots collected at 1-hour intervals comprise the data used for analysis of mineral deposition reported in this study.

Figure $3 A$ also shows that the mineral band position is timeindependent, or at least varies less than we can measure by band fitting (approximately $\pm 0.2 \mathrm{~cm}^{-1}$ ). Thus, our measurements are silent on the long-running discussion over the presence or absence of intermediate forms of mineral that precede hydroxyapatite formation. At most, we can say that such forms, if they exist, decay to an apatitic form in less time than the 1-hour interval between samples.

As noted in Fig. 1, the mineralization front gradually advances over time from the interparietal bone edge inward to the fontanel region. To determine if this advancing front could be detected by Raman measurements, time sequence spectra were taken at several points near the initial position interparietal bone edge. Measurements were taken along a line perpendicular to the edge (see Fig. $3 B$ ) at regular intervals of $25 \mu \mathrm{m}$ beginning within the mineralized region and extending into the fontanel. Figure $3 C$ shows complete spectra taken for each position at the beginning of the experiment, whereas Fig. 3D shows kinetic profiles of mineral deposition (mineral-to-matrix ratio) at each position with negative positions corresponding to regions within bone and positive positions to fontanel. At time zero, the $940 \mathrm{~cm}^{-1}$ collagen band and the $1003 \mathrm{~cm}^{-1}$ phenylalanine band, which at this point is predominantly from noncollagenous proteins, are seen in all spectra (Fig. $3 A$ ). The edge of the bone shows a small amount of the mineral band at $957 \mathrm{~cm}^{-1}$, but is barely resolvable from background. In the fontanel there is no discernible mineral band; but mineral is observed in the bone region away from the edge having a Raman spectrum with a well-defined $957 \mathrm{~cm}^{-1}$ band. Although the fontanel region showed no discernible mineral signal, we cannot rule out the possibility of trace mineral deposited prior to time zero. It is also not clear whether the presence of prior mineral has any effect on later mineral dynamics. Net mineral gain was consistently
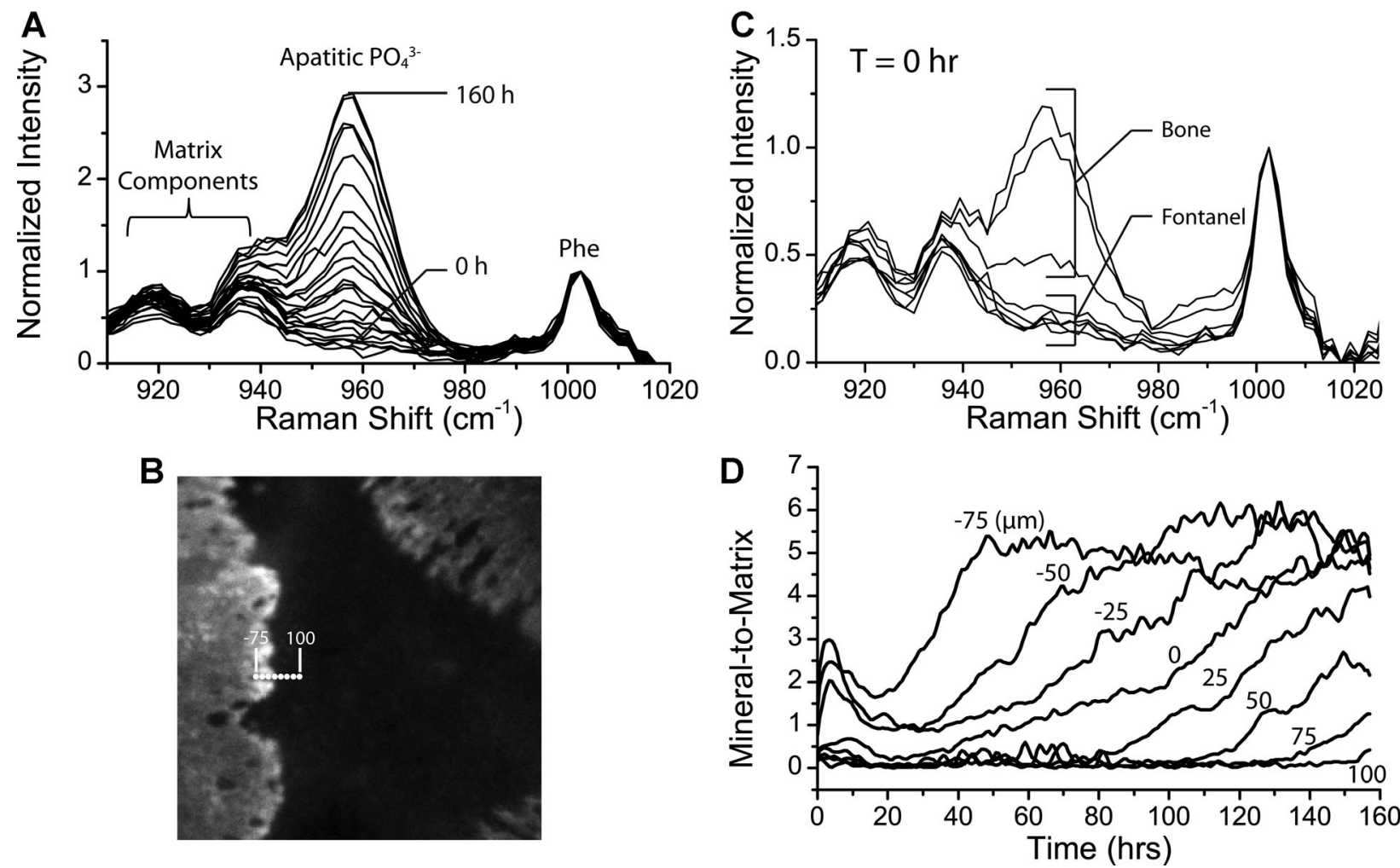

Fig. 3. Mineral deposition observed by Raman spectroscopy. ( $A$ ) Overlay of Raman spectra of the interparietal bone edge with 10-hour time intervals, normalized to the Phe band. $(B)$ Fluorescence image of a murine calvarium in culture at $\mathrm{T}=0$ with eight Raman probe positions near the interparietal bone edge indicated with white dots. (C) Overlay of Raman spectra at each probe position at $T=0$. $(D)$ Time-series data of mineral deposition with distances from the interparietal bone edge indicated. 
observed over time at each position. Those positions in the fontanel region showed delayed mineral deposition corresponding to the distance from the initial edge, which supports the model of inward bone growth into a previously unmineralized fontanel region.

\section{Circadian regulation of mineralization}

In a Raman study of 10 neonatal murine calvaria, the probe was positioned within $50 \mu \mathrm{m}$ of the interparietal bone/fontanel boundary (white arrowheads in Fig. $2 B$ ) on the unmineralized fontanel side, and mineralization was observed for 6 days with 1-hour time intervals. Raman spectra showed evidence of mineralization as an increase in the intensity of the bone mineral phosphate Raman signal $\left(957 \mathrm{~cm}^{-1}\right)$ through the course of the experiment. In six calvaria, circadian periodicity was clearly seen in mineral formation rate. Mineral deposition occurred in bursts followed by intervals of low deposition or no deposition. In the recorded data, mineralization is seen as stair-step increases of the mineral-to-matrix ratio over time (Fig. 4A). Mineralization rate was measured as the time-derivative (see Fig. $4 B$ ). Fluctuating mineralization resulted in peaks and troughs. We were able to observe between two and six bursts over the 6-day period. The average period measured as the peak-to-peak time was 26.8 \pm 9.6 hours. Specimens that displayed one or no mineral event were not included in the analysis.

To estimate the phase delay between Per 1 expression and increased mineral activity, we compared the times of the earliest maximum bioluminescence and maximum mineralization rate after sacrifice. Per 1 expression and mineralization were measured independently. The first maxima of Per1 expression across calvaria $(n=14)$ occurred at СТ09:59 \pm 03:37. The first mineralization event occurs at CT16:51 $\pm 03: 45$. This mineralization lags approximately 7 hours behind Per1 expression $(p=0.004)$. Figure 5 shows the relative times of Per 1 expression and mineralization in a 24-hour clock format. ${ }^{(37)}$

\section{Aperiodic changes in mineral crystallinity}

The first mineral detected along the interparietal bone edge spectroscopically resembles small apatitic crystallites. In the early hours of observation the mineral phosphate has a characteristic $\mathrm{PO}_{4}{ }^{3-}$ Raman band centered at $957 \mathrm{~cm}^{-1}$ (Fig. 3A). This band position is consistent with previous Raman studies on young bone, ${ }^{(29)}$ but contrasts with more mature bone mineral, which typically has a band centered at $959 \mathrm{~cm}^{-1}$. (36) The band center remains constant as mineral accumulates. However, crystallinity is initially $14 \pm 2.5 \mathrm{~nm}$ and increases almost linearly at an average rate of $0.068 \pm 0.021 \mathrm{~nm} / \mathrm{h}$ and then reaches a plateau at $21.7 \pm 2.6 \mathrm{~nm}$ with no further change (Fig. 4C). Similar aperiodic behavior was repeatedly observed across calvaria displaying periodic growth $(n=6)$. These results suggest that there is a crystallinity limit achievable in this tissue system, although it is not always reached within 6 days.

\section{Discussion}

Here we show the first direct evidence for circadian periodicity in mammalian bone mineralization. Neonatal murine calvarial
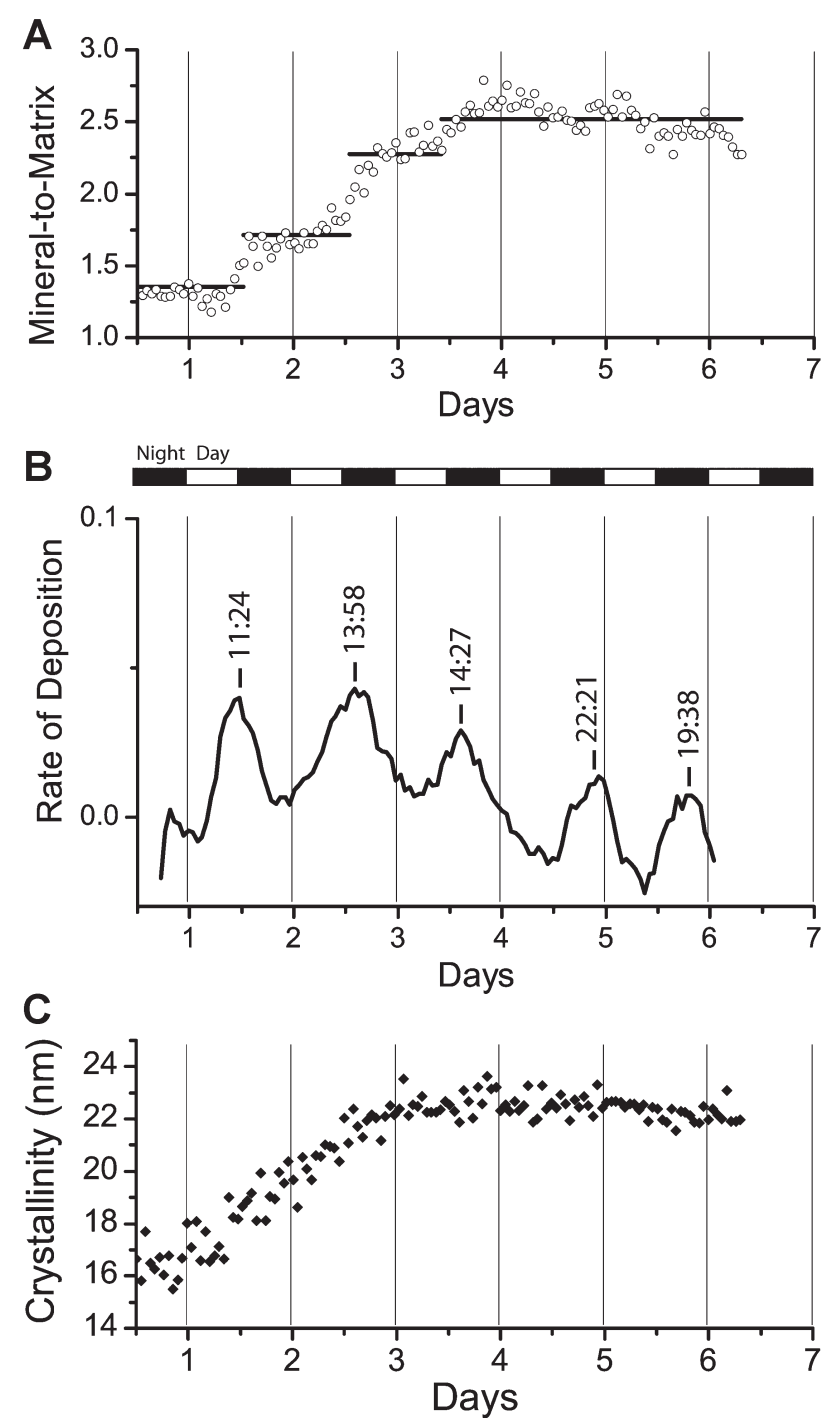

Fig. 4. Mineral deposition at the interparietal bone/fontanel boundary in ex vivo calvarial tissue measured by Raman spectroscopy. (A) Circadian time dependence of the mineral-to-matrix ratio (amount) with segmented line (solid) illustrating incremental increases in tissue mineral content. ( $B$ ) Circadian time dependence of deposition rate, with black and white bars indicating dark and light times during gestation. (C) Time dependence of mineral crystallinity.

organ cultures demonstrated in vitro mineralization and thereby facilitated the tracking of mineralization kinetics. Cyclic events were observed as pulses of heightened mineralization occurring periodically with an interval of approximately 1 day. Per 1 expression confirms the presence and operation of the clock genes functioning in calvarial tissue. In previous studies, circadian clock gene activity was observed for up to 30 days in SCN organ cultures, and peripheral clock function was detected for up to 7 days in isolated lung, liver, and skeletal muscle tissues. ${ }^{(14)}$ We observed circadian Per1 expression in calvarial organ cultures for up to 6 days, consistent with previous observations with other peripheral clocks in organ culture. Although circadian rhythms in bone formation has been inferred from fluctuating biomarkers and metabolites, periodicity in the biosynthesis of bone mineral has not previously been measured. 


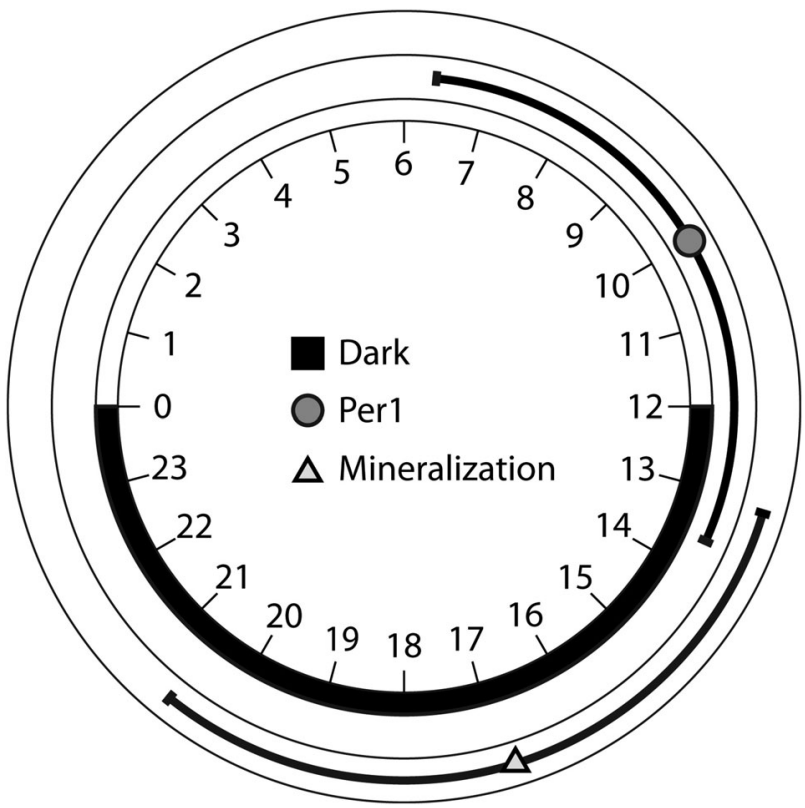

Fig. 5. Circular graphic showing circadian Per1 expression and bone mineralization. Circadian time, inner circle. Median Per 1 expression and mineralization times shown as dark gray circle and light gray triangle, with bars representing the standard deviation. Dark region indicates lights off time during gestation.

The timed expression of the Per 1 gene in murine calvarial tissue is consistent with previous reports of clock genes in other peripheral tissue. Because much of the mammalian clock mechanism has been elucidated, we can suggest how bone mineralization fits into the circadian process. In liver, Per 1 mRNA levels peak at CT10, with translation following at CT15 to CT18. ${ }^{(38)}$ After phosphorylation in the cytoplasm, PER proteins form a complex with CRY proteins, which suppresses BMAL:CLOCK transcription. Bmal is expressed as RNA at CT21 to CT03 and then translated to protein at CT15 to СT03. ${ }^{(38)}$ Recently, Koike and colleagues $^{(39)}$ showed that BMAL:CLOCK transcriptional activity peaks at CT15 and decreases at CT16 to CT20. In our study, maximum mineral deposition occurs from CT13 to CT22, strongly suggesting that mineralization is associated with BMAL:CLOCK transcriptional activity. Ours is the first study to directly demonstrate a correlation between peripheral clock operation and bone mineralization. Moreover, because the master clock in the SCN is not present, we have shown that circadian regulation of mineral deposition is an autonomous process in organ culture, although presumably synchronized by the master clock in intact organisms.

Development of mineral crystallinity does not appear to be directly regulated by a clock. Instead, small apatitic crystals continuously grow larger, at least along the crystallographic caxis. Mineral crystallinity increases approximately linearly with time before plateauing. Intermediate crystal phases such as amorphous calcium phosphate or octacalcium phosphate that have been proposed in more controlled systems were not detected. ${ }^{(28,40)}$ As reviewed by Gorski, ${ }^{(26,41)}$ the mineralization process can be considered as having two parts: (1) a nucleation phase in which crystallites of 15 to $20 \mathrm{~nm}$ in size are deposited into the extracellular matrix (ECM); and (2) a growth phase during which individual crystals increase in size. The first detected mineral in the present study had a crystallinity of $14 \mathrm{~nm}$. Because it forms within a previously unmineralized fontanel region adjacent to the mineralization front, this mineral must result from de novo nucleation. After several days of increasing crystallinity a plateau of about $22 \mathrm{~nm}$ was reached. Our experiments strongly suggest that the nucleation process is governed by circadian oscillatory signaling, but the increase in crystallite size is not, at least over the 5-day to 6-day durations of our measurements. For crystallites to propagate into the nonmineralized matrix both nucleation and crystal growth are required. We are observing both. However, we have no evidence about what protein or proteins mediate this process.

There is currently no consensus on a mechanism for limiting crystal growth to a certain size. Possibilities include the suggested timed expression of crystal growth inhibitors and the limited volume of type I collagen gap regions. ${ }^{(25,42)}$ However, crystal growth of apatites in vitro has also been shown to be limited by crystal ionic composition. ${ }^{(43)}$ Carbonate substitution destabilizes an apatitic crystal lattice and limits crystals to a maximum size. Regulation of mineral crystallinity may not involve direct interaction with proteins, but rather occurs through regulation of carbonate, phosphate, or calcium concentration in the ECM.

There are limitations to our study. Importantly, for every tissue specimen we chose a measurement region at the mineralization front at the start of an experiment. This same region is interrogated throughout the experiment, but the mineralization front continues to advance. Thus, at the first measurement time we may see mineral deposition on newly laid down matrix, but all subsequent measurements are of new mineral deposited on preexisting mineral. In short, as the experiment proceeds it becomes increasingly difficult to see any details of first mineral. Transient mineral intermediates, if any, are obscured by the earlier-deposited mineral.

Our experimental apparatus does not allow observation of mineral deposition and luminescence in the same experiment. Raman spectroscopy requires use of a high-magnification/high-NA objective to maximize observation of a thin layer of newly modeled mineral. But weak luminescence measurement requires a low-magnification objective to collect signal from a large area of the specimen. Although automation of change of objectives is possible, the instrumentation available for this study does not have this capability.

Although our results imply that any osteoblast-directed ossification is governed by circadian processes, current measurements were only made in calvarial intramembranous bone. We provide no direct evidence that circadian rhythms occur in endochondral ossification. We also do not know how circadian processes interact with genetic defects affecting the amount and quality of mineral. ${ }^{(44,45)}$ Our specimen immobilizer was designed for disk-shaped specimens. Completely different design would be needed for study of endochondral ossification in ribs or long bones. Even at its present state of development or with simple instrument modifications our model may be applicable to study of therapeutic interventions to bone resorption and formation that may disrupt the circadian processes. However, anabolic treatments that target osteoblast activity are more likely to disrupt circadian rhythms in osteogenesis. Luchavova and colleagues $^{(46)}$ showed that once-daily injections of teriparatide 
(PTH(1-34)) induced twice-daily peaks in bone turnover markers. A less frequent dose is required for treatment with sclerostin antibody; however, its effect on circadian mineralization has not been determined. Anticatabolic treatment with bisphosphonates in rats reduces the daily resorption of bone, but shows no sign of perturbing the circadian pattern and has no effect on bone formation marker magnitude or frequency. ${ }^{(47)}$ However, it is not known if the lack of Haversian systems in this lower bone turnover animal model would affect the interpretation of this result.

In summary, this study provides further details of the circadian rhythm of bone formation including evidence for oscillatory behavior of mineralization. Based on the current evidence we see no other explanation for periodic behavior in these calvarial explants than the intervention of a local circadian mechanism in intramembranous ossification, particularly in the nucleation of apatitic mineral. It is not clear at this point whether circadian signaling also plays a role in mineral propagation and crystallinity; however, the data here suggest no influence. Because our measurements are made on ex vivo specimens, it remains possible that diurnal synchronization by the master clock will affect details of our findings if such measurement were to be made on live animals.

\section{Disclosures}

All authors state that they have no conflicts of interest.

\section{Acknowledgments}

This work was supported by NIH grant RO1 AR047969. JDM acknowledges support through training grant T90 DK070071. CW was supported by the training grant T32 DE007057. We acknowledge the assistance of Dr. Francis Esmonde-White for technical advice on designing the Raman microscope, and Matthew Kole for hands on assistance in acquiring data. We also thank Steven Donajkowski for designing and machining the tissue immobilization device used in this study.

Authors' roles: Study design: JDM, GZ, RF, and MM. Data collection and analysis: JDM, GZ, AK, and CW. Data interpretation: JDM, GZ, AK, CW, RF, and MM. Instrument design: JDM and AK. Drafting manuscript: JDM. Revising and Approving manuscript content: JDM GZ, AK, CW, RF, and MM. JDM and GZ take responsibility for the integrity of the data analysis.

\section{References}

1. Kwon I, Choe HK, Son GH, Kim K. Mammalian molecular clocks. Exp Neurobiol. 2011;20(1):18-28.

2. Reppert SM, Weaver DR. Coordination of circadian timing in mammals. Nature. 2002;418(6901):935-41.

3. Balsalobre A, Brown SA, Marcacci L, Tronche F, Kellendonk C, Reichardt HM, Schutz G, Schibler U. Resetting of circadian time in peripheral tissues by glucocorticoid signaling. Science. 2000;289 (5488):2344-7.

4. Maywood ES, O'Neill JS, Chesham JE, Hastings MH. Minireview: The circadian clockwork of the suprachiasmatic nuclei-analysis of a cellular oscillator that drives endocrine rhythms. Endocrinology. 2007;148(12):5624-34.
5. Schibler U, Sassone-Corsi P. A web of circadian pacemakers. Cell. 2002;111(7):919-22.

6. Mohawk JA, Green CB, Takahashi JS. Central and peripheral circadian clocks in mammals. Annu Rev Neurosci. 2012;35:445-62.

7. Lee C, Etchegaray JP, Cagampang FR, Loudon AS, Reppert SM. Posttranslational mechanisms regulate the mammalian circadian clock. Cell. 2001;107(7):855-67.

8. Kume K, Zylka MJ, Sriram S, Shearman LP, Weaver DR, Jin X, Maywood ES, Hastings $\mathrm{MH}$, Reppert SM. mCRY1 and mCRY2 are essential components of the negative limb of the circadian clock feedback loop. Cell. 1999;98(2):193-205.

9. Duong HA, Robles MS, Knutti D, Weitz CJ. A molecular mechanism for circadian clock negative feedback. Science. 2011;332(6036):1436-9.

10. Maronde E, Schilling AF, Seitz S, Schinke T, Schmutz I, van der Horst G, Amling M, Albrecht $U$. The clock genes Period 2 and Cryptochrome 2 differentially balance bone formation. PLOS One. 2010;5(7):e11527.

11. Zvonic S, Ptitsyn AA, Kilroy G, Wu X, Conrad SA, Scott LK, Guilak F, Pelled G, Gazit D, Gimble JM. Circadian oscillation of gene expression in murine calvarial bone. J Bone Miner Res. 2007;22(3):357-65.

12. Gafni Y, Ptitsyn AA, Zilberman Y, Pelled G, Gimble JM, Gazit D. Circadian rhythm of osteocalcin in the maxillomandibular complex. J Dent Res. 2009;88(1):45-50.

13. Fu L, Patel MS, Bradley A, Wagner EF, Karsenty G. The molecular clock mediates leptin-regulated bone formation. Cell. 2005;122(5):803-15.

14. Yamazaki S, Numano R, Abe M, Hida A, Takahashi R, Ueda M, Block GD, Sakaki Y, Menaker M, Tei H. Resetting central and peripheral circadian oscillators in transgenic rats. Science. 2000;288(5466):682-5.

15. Lamia KA, Storch KF, Weitz CJ. Physiological significance of a peripheral tissue circadian clock. Proc Natl Acad Sci U S A. 2008;105 (39):15172-7.

16. Storch KF, Paz C, Signorovitch J, Raviola E, Pawlyk B, Li T, Weitz CJ. Intrinsic circadian clock of the mammalian retina: importance for retinal processing of visual information. Cell. 2007;130(4):730-41.

17. Zvonic S, Ptitsyn AA, Conrad SA, Scott LK, Floyd ZE, Kilroy G, Wu X, Goh BC, Mynatt RL, Gimble JM. Characterization of peripheral circadian clocks in adipose tissues. Diabetes. 2006;55(4):962-70.

18. Duffield GE, Best JD, Meurers BH, Bittner A, Loros JJ, Dunlap JC. Circadian programs of transcriptional activation, signaling, and protein turnover revealed by microarray analysis of mammalian cells. Curr Biol. 2002;12(7):551-7.

19. Balsalobre A, Damiola F, Schibler U. A serum shock induces circadian gene expression in mammalian tissue culture cells. Cell. 1998;93 (6):929-37

20. Edery I. A master CLOCK hard at work brings rhythm to the transcriptome. Genes Dev. 2011;25(22):2321-6.

21. Zhang EE, Kay SA. Clocks not winding down: unravelling circadian networks. Nat Rev Mol Cell Biol. 2010;11(11):764-76.

22. Mengatto CM, Mussano F, Honda Y, Colwell CS, Nishimura I. Circadian rhythm and cartilage extracellular matrix genes in osseointegration: a genome-wide screening of implant failure by vitamin D deficiency. PLOS One. 2011;6(1):e15848.

23. Hanyu R, Hayata T, Nagao M, Saita Y, Hemmi H, Notomi T, Nakamoto T, Schipani E, Knonenbery H, Kaneko K, Kurosawa H, Ezura Y, Noda M. Per-1 is a specific clock gene regulated by parathyroid hormone (PTH) signaling in osteoblasts and is functional for the transcriptional events induced by PTH. J Cell Biochem. 2011;112(2):433-8.

24. Gundberg CM, Markowitz ME, Mizruchi M, Rosen JF. Osteocalcin in human serum: a circadian rhythm. J Clin Endocrinol Metab. 1985;60 (4):736-9.

25. Hunter GK, Hauschka PV, Poole AR, Rosenberg LC, Goldberg HA, Nucleation and inhibition of hydroxyapatite formation by mineralized tissue proteins. Biochem J. 1996;317(Pt 1):59-64.

26. Gorski JP. Biomineralization of bone: a fresh view of the roles of noncollagenous proteins. Front Biosci. 2012;17:2598-621. 
27. Colfen $\mathrm{H}$, Biomineralization: a crystal-clear view. Nat Mater. 2010;9:960-1.

28. Nudelman F, Pieterse K, George A, Bomans PHH, Friedrich H, Brylka LJ, Hilbers PAJ. With Gd, Sommerdijk NAJM. The role of collagen in bone apatite formation in the presence of hydroxyapatite nucleation inhibitors. Nat Mater. 2010;9:1004-9.

29. Crane NJ, Popescu V, Morris MD, Steenhuis P, Ignelzi JMA. Raman spectroscopic evidence for octacalcium phosphate and other transient mineral species deposited during intramembranous mineralization. Bone. 2006;39(3):434-42.

30. Weiner S. Transient precursor strategy in mineral formation of bone. Bone. 2006;39(3):431-3.

31. Grynpas MD, Omelon S. Transient precursor strategy or very small biological apatite crystals?. Bone. 2007;41(2):162-4.

32. Wilsbacher LD, Yamazaki S, Herzog ED, Song EJ, Radcliffe LA, Abe M, Block G, Spitznagel E, Menaker M, Takahashi JS. Photic and circadian expression of luciferase in mPeriod1-luc transgenic mice in vivo. Proc Natl Acad Sci U S A. 2002;99(1):489-94.

33. Wang D, Christensen K, Chawla K, Xiao G, Krebsbach PH, Franceschi RT. Isolation and characterization of MC3T3-E1 preosteoblast subclones with distinct in vitro and in vivo differentiation/mineralization potential. J Bone Miner Res. 1999;14(6):893-03.

34. Li Y, Ge C, Franceschi RT. Differentiation-dependent association of phosphorylated extracellular signal-regulated kinase with the chromatin of osteoblast-related genes. J Bone Miner Res. 2010;25 (1):154-63.

35. Press WH, Teukolsky SA, Vetterling WT, Flannery BP. Numerical recipes in C. 2nd ed. New York: Cambridge University Press; 1992.

36. McElderry J-DP, Kole MR, Morris MD. Repeated freeze-thawing of bone tissue affects Raman bone quality measurements. J Biomed Optics. 2011;16(7):071407.

37. Relogio A, Westermark PO, Wallach T, Schellenberg K, Kramer A, Herzel $H$. Tuning the mammalian circadian clock: robust synergy of two loops. PLoS Comput Biol. 2011;7(12):e1002309.
38. Lowrey PL, Takahashi JS. Mammalian circadian biology: elucidating genome-wide levels of temporal organization. Annu Rev Genomics Hum Genet. 2004;5:407-1.

39. Koike N, Yoo SH, Huang HC, Kumar V, Lee C, Kim TK, Takahashi JS. Transcriptional architecture and chromatin landscape of the core circadian clock in mammals. Science. 2012;338(6105):349-54.

40. Kazanci M, Fratzl P, Klaushofer K, Paschalis EP. Complementary information on in vitro conversion of amorphous (precursor) calcium phosphate to hydroxyapatite from Raman microspectroscopy and wide-angle X-ray scattering. Calcif Tissue Int. 2006;79(5):354-9.

41. Gorski JP. Is all bone the same? Distinctive distributions and properties of non-collagenous matrix proteins in lamellar vs. woven bone imply the existence of different underlying osteogenic mechanisms. Crit Rev Oral Biol Med. 1998;9(2):201-23.

42. Bonucci E. Bone mineralization. Front Biosci. 2012;17:100-28.

43. Baig AA, Fox JL, Young RA, Wang Z, Hsu J, Higuchi WI, Chhettry A, Zhuang $H$, Otsuka M. Relationships among carbonated apatite solubility, crystallite size, and microstrain parameters. Calcif Tissue Int. 1999;64(5):437-49.

44. Camacho NP, Hou L, Toledano TR, Ilg WA, Brayton CF, Raggio CL, Root $\mathrm{L}$, Boskey AL. The material basis for reduced mechanical properties in oim mice bones. J Bone Miner Res. 1999;14(2):264-72.

45. Wojtowicz A, Dziedzic-Goclawska A, Kaminski A, Stachowicz W, Wojtowicz K, Marks SC Jr, Yamauchi M. Alteration of mineral crystallinity and collagen cross-linking of bones in osteopetrotic toothless $(\mathrm{t} / \mathrm{t} \mathrm{l})$ rats and their improvement after treatment with colony stimulating factor-1. Bone. 1997;20(2):127-32.

46. Luchavova M, Zikan V, Michalska D, Raska I Jr, Kubena AA, Stepan JJ. The effect of timing of teriparatide treatment on the circadian rhythm of bone turnover in postmenopausal osteoporosis. Eur J Endocrinol. 2011;164(4):643-8.

47. Shao $P$, Ohtsuka-Isoya $M$, Shinoda $H$. Circadian rhythms in serum bone markers and their relation to the effect of etidronate in rats. Chronobiol Int. 2003;20(2):325-36. 